\title{
Génétique du canal sodium épithélial : nouveaux développements dans la compréhension du contrôle de la pression sanguine et de la réabsorption de liquide dans les voies aériennes et le poumon
}

Le canal sodium épithélial, sensible à l'amiloride $(\mathrm{ENaC})$ est l'étape clé de la réabsorption électrogénique de sodium luminal dans la partie distale du néphron, dans la partie distale du côlon et dans les voies aériennes supérieures et distales. La structure primaire de ce canal ionique, très sélectif pour le sodium a été récemment publiée [1]. Il s'agit d'une protéine multimérique, composée de trois sous-unités homologues, appelées $\alpha \beta$ et $\gamma$. Le gène du canal sodium épithélial fait partie d'une nouvelle super-famille de gènes qui codent pour des canaux ioniques impliqués dans le contrôle du volume cellulaire et extracellulaire, dans la transmission synaptique [2] et dans le contrôle de fonctions telles que le goût [3] ou le toucher [4] $\left(\mathrm{m} / \mathrm{s}^{\circ} 3\right.$, vol. $10, p$. 365). Jusque récemment, les conséquences physiopathologiques de cette découverte n'avaient pu être appréciées à leur juste valeur par manque de sondes spécifiques pour détecter des mutations dans les gènes des trois sous-unités et/ou pour mesurer le taux d'expression de ces gènes dans les organes tels que le rein, le côlon ou le poumon. Il semble bien maintenant qu'à la lumière de récentes découvertes, le rôle de ce canal ionique dans la physiopathologie humaine va devenir plus important que l'on avait pu le croire au premier abord. Cette impression vient d'études génétiques: d'abord, les investigations de plusieurs mala- dies monogéniques chez l'homme impliquant des désordres de l'équilibre sodique et, ensuite, le développement récent d'un modèle animal chez la souris, où la sous-unité $\alpha d u$ canal sodique a été inactivée, sousunité indispensable pour l'activité du canal dans la membrane (figure 1).

Le syndrome de Liddle est une maladie monogénique autosomique dominante qui se manifeste par une hypertension sensible au sel (pseudohyperaldostéronisme). Dans ce cas, des mutations de type délétion ou des mutations ponctuelles dans l'extrémité carboxy-terminale des sous-unité $\beta$ et $\gamma$ de ENaC ont été décrites $\left(m / s n^{\circ} 2\right.$, vol. $11, p$. 296) [5-7]. Quand ces mutations sont exprimées dans l'ovocyte de Xenopus, elles conduisent à un phénotype de canal hyperactif suggérant que, chez l'homme, on a affaire à des mutations conduisant à un "gain» de fonction ou, du moins, à une altération de la régulation du canal sodium [8-10]. Des expériences de mutagenèse systématique de cette région carboxy-terminale, et la comparaison avec d'autres protéines [11] ont conduit récemment à proposer l'existence d'un motif consensus PPxY (Pro-Pro-X-Tyr) qui aurait un rôle essentiel dans les altérations responsables du phénotype Liddle [11]. Ce motif est délété dans les troncations qui sont la cause de plusieurs génotypes Liddle et, dans deux familles, une seule mutation ponctuelle touchant directement ce motif est suffisante pour produire la maladie $[6,12]$.

A l'heure actuelle, nous postulons que cette région est capable d'interaction protéine-protéine et un laboratoire canadien, à Toronto [13], a récemment démontré que cette même région de la protéine peut lier une protéine appelée NEDD-4 [13]. L'hypothèse actuelle est que cette protéine est un répresseur de l'activité du canal; lorsque la région d'interaction est mutée ou délétée, le canal ne peut plus lier le répresseur et devient hyperactif.

Très récemment [14], des mutations conduisant à la perte de fonction du canal ont été décrites dans quelques familles humaines souffrant d'un syndrome de perte de sel (pseudohypoaldostéronisme de type 1). A la naissance, ces enfants deviennent rapidement hypovolémiques, souffrent d'une acidose métabolique et d'hyperkaliémie sévère. La mort peut s'ensuivre rapidement si les pertes de sel ne sont pas compensées et si l'hyperkaliémie n'est pas jugulée. Trois types de mutations ont été décrits par le groupe de Lifton à Yale (New Haven, USA) ; dans deux cas il s'agit de délétions importantes de la sous-unité $\alpha$ de ENaC et, dans un troisième cas, d'une mutation ponctuelle, d'une glycine en sérine dans le la région amino-terminale de la sous-unité $\beta$. Quand cette mutation est exprimée dans le système d'ex- 


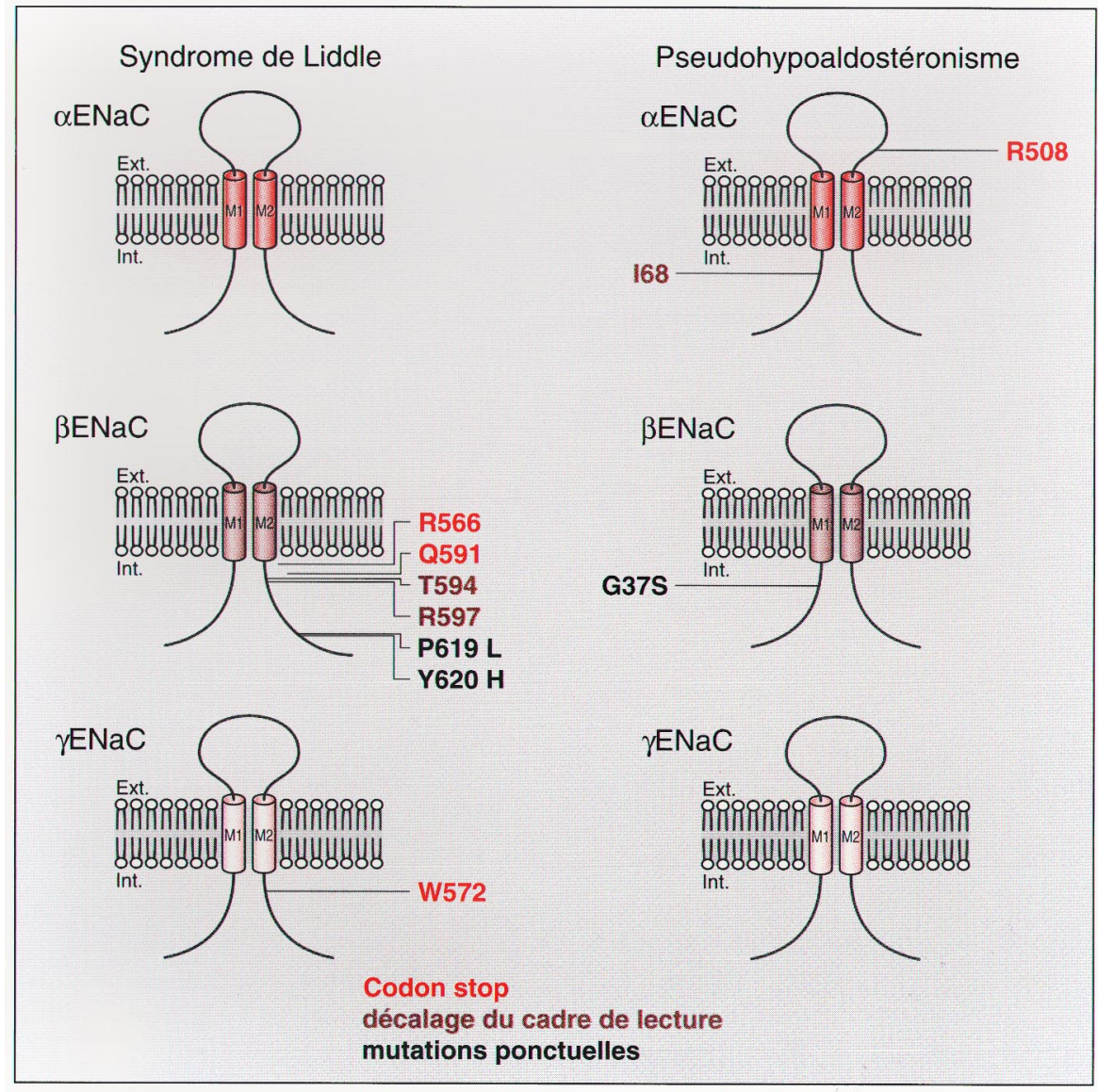

Figure 1. Les trois sous-unités du canal sodium épithélial (ENaC) et leurs mutations. Des mutations dans la partie carboxyterminale des sous-unités $\beta$ et $\gamma$ rendent le canal hyperactif et sont la cause de plusieurs génotypes Liddle d'hypertension artérielle; ce gain de fonction pourrait naître de la suppression de l'interaction avec une protéine inhibitrice (NEDD-4). A l'inverse, des mutations entraînant une perte de fonction ont été décrites dans la partie aminoterminale des sous-unités $\alpha$ et $\beta$, responsables d'un syndrome de perte de sel (pseudohypoaldostéronisme). L'invalidation du gène codant pour la sous-unité $\alpha$ de ENaC a montré le rôle majeur joué par ce canal dans la réabsorption néonatale du liquide alvéolaire et fait envisager que certains syndromes de détresse respiratoire du prématuré seraient liés à l'immaturité de ce canal sodique.

pression de l'ovocyte, on observe une diminution mais pas une perte complète de l'activité d'ENaC [14]. Finalement, Edith Hummler et al. (Lausanne, Suisse) [15] ont récemment obtenu une souris dont les allèles codant pour la sous-unité $\alpha$ de $\mathrm{ENaC}$ ont été invalidés. Les résultats de cette mutation sont spectaculaires. Le transport de sodium électrogénique, sensible à l'amiloride, est supprimé dans les voies aériennes de la souris; les souris homozygotes $E N a C^{-/}$développent un syndrome tion pulmonaire satisfaisante par l'administration de surfactants mais, dans d'autres cas, le syndrome de détresse respiratoire résiste à ce traitement. Nous proposons que l'immaturité du canal sodique chez le nouveau-né pourrait être un facteur aggravant du syndrome de détresse respiratoire. D'autres affections (cardiaques, neurologiques) pourraient être liées à des mutations d'autres membres de la famille $\mathrm{ENaC}$ exprimés surtout dans le cour et le cerveau [16].

Les descriptions de ces «canalopathies " du canal sodique devraient stimuler de nouvelles recherches dans le domaine du contrôle de la pression sanguine et des syndromes de détresse respiratoire et de l'œdème du poumon. Du point de vue thérapeutique, cela ouvre de nouvelles perspectives de développement de médicaments qui pourraient, soit bloquer le canal sodique dans le cas d'hypertension sensible au sel, soit, au contraire, activer le canal sodique dans le cas de détresse respiratoire.

B.C.R.

E.H.

J.D.H.

L.S.

1. Canessa CM, Schild L, Buell G, Thorens B, Gautschi I, Horisberger JD, Rossier BC. The amiloride-sensitive sodium channel is made of three homologous subunits. Nature 1994; 367 : 463-7. 2. Lingueglia E, Champigny G, Lazdunski M, Barbry $\mathrm{P}$. Cloning of the amiloride-sensitive FMRFamide peptide-gated sodium channel. Nature 1995 ; 378: 730-3.

3. Li XJ, Xu RH, Guggino WB, Snyder SH. Alternatively spliced forms of the $\alpha$ subunit of the epithelial sodium channel: distinct sites for amiloride binding and channel pore. Mol Pharmacol $1995 ; 47$ : 1133-40.

4. Chalfie, M. Touch receptor development and function in Caenorhabditis elegans. J Neurobiol 1993 ; 24: 1433-41.

5. Shimkets RA, Warnock DG, Bositis CM, Nelson Williams C, Hansson JH, Schambelan M, Gill Jr JR, Ulick S, Milora RV, Findling JW, Canessa CM, Rossier BC, Lifton RP. Liddle's syndrome: heritable human hypertension caused by mutations in the $\beta$ subunit of the epithelial sodium channel. Cell 1994; 79 : 407-14.

6. Hansson JH, Schild L, Lu Y, Wilson TA, Gautschi I, Shimkets R, Nelson-Williams C, Rossier BC, Lifton RP. A de novo missense mutation of the $\beta$ subunit of the epithelial sodium channel causes hypertension and Liddle's syndrome and identifies a proline-rich segment of the protein critical for regulation of channel activity. Proc Natl Acad Sci USA 1995; 92: 11495-9. 
7. Hansson JH, Nelson-Williams C, Suzuki H, Schild L, Shimkets R, Lu Y, Canessa CM, Iwasaki T, Rossier BC, Lifton RP. Hypertension caused by a truncated epithelial sodium channel $\gamma$ subunit: genetic heterogeneity of Liddle syndrome. Nature Genet 11995; 1 : 76-82.

8. Schild L, Canessa CM, Schimkets RA, Gautschi I, Lifton RP, Rossier BC. A mutation in the epithelial sodium channel causing Liddle disease increases channel activity in the Xenopus laevis oocyte expression system. Proc Natl Acad Sci USA 1995; 92: 5699-703.

9. Snyder PM, Price MP, McDonald FJ, Adams CM, Volk KA, Zeiher BG, Stokes JB, Welsh MJ. Mechanism by which Liddle's syndrome mutations increase activity of a human epithelial $\mathrm{Na}^{+}$ channel. Cell 1995; 83: 969-78.

10. Schild L, Lu Y, Gautschi I, Schneeberger E, Lifton RP, Rossier BC. Identification of a PY motif in the epithelial Na channel subunit as a target sequence for mutations causing channel activation found in Liddle syndrome. EMBO $J$ 1996; 15 : 2381-7.

11. Einbond A, Sudol M. Towards prediction of cognate complexes between the WW domain and proline-rich ligands. FEBS Lett 1996 (sous presse). 12. Tamura H, Schild L, Enomoto N, Matsui N, Marumo F, Rossier BC, Sasaki S. Liddle disease caused by a missense mutation of $\beta$ subunit of the epitehlial sodium channel gene. J Clin Invest 1996 (sous presse).

13. Staub O, Dho S, Henry P, Correa J, Ishikawa T, McGlade J, Rotin D. WW domains of NEDD4 bind to the proline rich regions in the epithelial $\mathrm{Na}^{+}$channel deleted in Liddle's syndrome. EMBO J 1996; 15: 2371-80.

14. Chang SS, Grunder S, Hanukoglu A, Rösler A, Mathew PM, Hanukoglu I, Schild L, Lu Y, Shimkets RA, Nelson-Williams C, Rossier BC, Lifton RP. Mutations in subunits of the epithelial sodium channel cause salt wasting with hyperkalaemic acidosis, pseudohypoaldosteronism type 1 . Nature Genet 1996; 12: 248-53.

15. Hummler E, Barker P, Gatzi J, Beermann F, Verdumo C, Schmidt A, Boucher R, Rossier BC. Early death due to defective neonatal lung liquid clearance in $\alpha \mathrm{ENaC}$-deficient mice. Nature Genet $1996 ; 12: 325-8$

16. Waldmann R, Champigny G, Bassilana F, Voilley N, Lazdunski M. Molecular cloning and functional expression of a novel amiloride-sensitive $\mathrm{Na}^{+}$channel. J Biol Chem 1995; 270 : 27411-4. 